\title{
Impact of changes in psychological resilience during treatment with intensity-modulated radiotherapy on nasopharyngeal carcinoma patients: a prospective study
}

\author{
$\mathrm{Ni} \mathrm{Lu}{ }^{1}$, Ting Qin ${ }^{1}$, Wenqing $\mathrm{Hu}^{2}$ \\ ${ }^{1}$ Ear, Nose, and Throat Department, the Affiliated Hospital of Jiangnan University, Wuxi, China; ${ }^{2}$ Department of Surgery, the Affiliated Hospital of \\ Jiangnan University, Wuxi, China \\ Contributions: (I) Conception and design: All authors; (II) Administrative support: W Hu; (III) Provision of study materials or patients: N Lu, T Qin; \\ (IV) Collection and assembly of data: N Lu, T Qin; (V) Data analysis and interpretation: N Lu; (VI) Manuscript writing: All authors; (VII) Final \\ approval of manuscript: All authors. \\ Correspondence to: Wenqing Hu. Department of Surgery, the Affiliated Hospital of Jiangnan University, No. 1000 Hefeng Road, Wuxi 214125 , China. \\ Email: wykhwq@163.com.
}

Background: Nasopharyngeal carcinoma (NPC) patients can undergo changes in psychological status
during treatment. The aim of this prospective study was to determine the impact of the changes in
psychological resilience on the quality of life (QoL) and long-term outcomes of patients.

Methods: Patients with NPC receiving intensity-modulated radiotherapy (IMRT) between March 2017 and February 2019 were prospectively included. Their psychological resilience was evaluated by the ConnorDavidson resilience scale (CD-RISC) twice. Patients were then divided into the improved psychological resilience group and the deteriorated group. All patients were followed up for at least 2 years, and acute or late severe complications were recorded. The QoL of patients was evaluated within 1 year by the European Organization for Research and Treatment of Cancer Quality of Life Questionnaire-Core 30-questions (QLQ-C30) and the Head and Neck 35-questions (HN35). Logistic regression analysis was used for the analysis of risk factors of psychological resilience in NPC patients. Similarly, linear regression analysis was used for the analysis of risk factors of QoL in NPC patients. The overall survival rate and progression-free survival rate were recorded and compared between the 2 groups using Kaplan-Meier curves.

Results: A total of 180 patients were included. The mean CD-RISC scores before radiotherapy and after radiotherapy were $55.8 \pm 7.0$ and $58.4 \pm 7.8$ points, respectively. Patients were divided into 104 patients in the improved group and 76 patients in the deteriorated group. Older age, advanced stage, chemotherapy treatment, and severe complications were important risk factors according to the multivariable logistic regression analysis. There were no significant differences in QLQ-C30 and HN35 scores before radiotherapy between the 2 groups, while significant differences were found in most items in the QLQ-C30 and HN35 between the 2 groups. Deteriorated resilience was identified as an important risk factor of QoL according to the multivariable linear regression analysis. NPC patients had significantly higher overall survival and progression-free survival in the improved group than in the deteriorated group.

Conclusions: Psychological resilience has an important impact on the prognosis of NPC patients, thus more attention should be paid to their psychological status during treatment with radiotherapy.

Keywords: Psychological resilience; intensity-modulated radiotherapy; nasopharyngeal carcinoma (NPC); prospective study

Submitted Nov 10, 2021. Accepted for publication Dec 24, 2021.

doi: 10.21037/apm-21-3592

View this article at: https://dx.doi.org/10.21037/apm-21-3592 


\section{Introduction}

Nasopharyngeal carcinoma (NPC) is a common malignant epithelial tumor of the head and neck in Southeast Asia, especially in southern China (1-3). Recent studies have reported that the incidence rate of NPC in southern China can reach up to 80 cases per 100,000 people (4). Worldwide, it was reported that there were 129,079 new NPC cases, and 72,987 patients died of NPC in 2018 (5). Due to the particular location of NPC, routine surgical treatment cannot be performed in NPC patients. At present, the most important treatment for NPC is radiation therapy, as NPC cells usually have high sensitivity to radiation (6). Meanwhile, the treatment site of radiotherapy is relatively limited, which can reduce the damage of radiation to other parts of body. For advanced NPC, the latest National Comprehensive Cancer Network guidelines also recommend early use of induction chemotherapy on the basis of radiotherapy or concurrent radiochemotherapy to improve the prognosis of patients (7).

The most widely used method of radiotherapy for NPC patients is intensity-modulated radiotherapy (IMRT), which is an improved form of traditional 3-dimensional (3D) conformal radiotherapy (8). The radiation dose can be more accurate in IMRT by adjusting the radiation intensity according to the $3 \mathrm{D}$ shape of the tumor. IMRT can also focus and apply a higher radiation dose to the area within the tumor, so that the surrounding normal tissue can receive the minimum radiation dose. Many studies have revealed that IMRT can more effectively kill NPC cells, reduce toxic reactions, improve prognosis, and prolong the survival duration of patients compared with traditional $2 \mathrm{D}$ radiotherapy (9-12). Compared with traditional 3D conformal radiotherapy, IMRT also has some advantages in reducing toxic reactions $(12,13)$. Therefore, IMRT has been widely used in the treatment of NPC patients.

Most previous studies have focused on the survival duration and tumor recurrence of NPC patients, while the change in the psychological status of NPC patients during treatment has received little attention. Generally, NPC patients will be impacted when they are diagnosed and during the process of treatment, leading to the emergence of negative emotions. A previous Chinese study showed that NPC patients had more physical complaints within 1 year of treatment, and it was difficult to alleviate them (14). Another study found that the most common psychological status of NPC patients included depression, anxiety, and fatigue, and the occurrence of these states could significantly reduce the quality of life (QoL) of patients (15). However, aside from the few above-mentioned studies, there is still a lack of research on the psychological status of NPC patients, which limits more in-depth psychological nursing of patients.

This prospective study focused on the changes in psychological resilience in NPC patients during IMRT treatment. Psychological resilience refers to the psychological and behavioral reaction of individual to the changed external environment. Psychological resilience not only reflects the emotional reactions of patients, but also the willingness of patients to overcome difficulties. The aim of this study was to determine the impact of changes in psychological resilience on the $\mathrm{QoL}$ and long-term outcomes of patients. In addition, the risk factors of psychological resilience in NPC patients were also calculated. We present the following article in accordance with the STROBE reporting checklist (available at https://apm.amegroups.com/article/view/10.21037/apm21-3592/rc).

\section{Methods}

\section{Patients}

All procedures performed in this study involving human participants were in accordance with the Declaration of Helsinki (as revised in 2013). The study was approved by institutional review board of the Affiliated Hospital of Jiangnan University (No. 2016033) and informed consent was taken from all the patients. Patients who met the following criteria were eligible for this study: (I) patients diagnosed with non-distant NPC; (II) patients admitted to our hospital for treatment between March 2017 and February 2019; and (III) patients receiving IMRT with or without chemotherapy. Patients were excluded from this study based on the following criteria: (I) patients younger than 18 years or older than 80 years; (II) patients receiving a variety of radiotherapy methods, including IMRT; (III) patients with psychiatric disorders or cognitive impairment; (IV) patients refusing to participate in this study or provided incomplete data; (V) patients with severe organ dysfunction; and (VI) patients with primary tumors in other parts of the body.

\section{IMRT}

IMRT was performed according to the protocol of our institution. Patients were placed in the supine position with fixation of the head and neck. Six-MV X-rays were 
used for IMRT. The gross tumor volume (GTV) included all tumors which were determined according to diagnostic CT or/and MRI, and the clinical target volume (CTV) included the GTV plus a 0.5 to $1 \mathrm{~cm}$ margin to encompass any microscopic extension. The irradiation dose was limited by the general condition of the NPC patient. Generally, the dose of GTV was set from 60 to $70 \mathrm{~Gy}$, and the dose of CTV from 50 to $60 \mathrm{~Gy}$. The fractional dose was set as 1.8 2.2 Gy per day, 5 days per week. The dose at the primary site and fractional dose were collected by investigators independently. In addition, induction or concurrent chemotherapy with a cisplatin-based regimen was also given to NPC patients for the treatment of bulky gross tumors or recurrent tumors.

\section{Psychological resilience}

The psychological resilience of the included patients was evaluated by the Connor-Davidson resilience scale (CDRISC). The CD-RISC contains 25 questions, with a maximum of 4 points and a minimum of 0 points for each question. The total score of the CD-RISC was 100 points. The psychological resilience of patients was evaluated from 3 dimensions: tenacity, self-strength, and optimism. The higher the total score, the better the patient's psychological resilience. The psychological resilience of the included patients was evaluated and recorded at least 2 times. The first time was at the first admission of the included NPC patients and the second time was at the end of IMRT. According to the changes in CD-RISC scores, the patients were divided into 2 groups: one group was the improved psychological resilience group (the second score was higher than the first score) and the other group was the deteriorated psychological resilience group (the second score was not higher than the first score).

\section{Data}

The following data were collected by 3 investigators independently: demographic data including age, gender, body mass index (BMI), marital status, educational level, histological type of NPC (type I, II, or III according to the classification of the World Health Organization), disease duration, clinical staging, the use of induction chemotherapy or concurrent radiochemotherapy, IMRT dose at the primary site, fractional dose, smoking history, and history of chronic disease, including hypertension, diabetes mellitus, chronic heart disease, chronic lung disease, and chronic kidney disease.

\section{Follow-up}

All included patients were followed up for at least 2 years after the end of IMRT, and all acute (within 6 months) or late severe complications (6 months later) related to radiotherapy were recorded. Most importantly, the QoL of included NPC patients was evaluated within 1 year using the European Organization for Research and Treatment of Cancer Quality of Life Questionnaire-Core 30-questions (QLQ-C30) and the Head and Neck 35-questions (HN35). The QLQ-C30 has been translated into Chinese and contains 5 functional items, 3 symptom items, 6 single items, and 1 general health item. The HN35 is a sitespecific questionnaire and contains 7 multiple items and 6 single items. Both the QLQ-C30 and HN35 have a total score of 100 points and a higher score represents better QoL. The overall survival rate and progression-free survival rate were also recorded during the follow-up.

\section{Statistical analysis}

Continuous variables were expressed as mean \pm standard deviation and analyzed using Student's $t$-test. Categorical variables were expressed as number (percentage) and analyzed using the chi-square test. Univariable and multivariable linear regression analysis was used for the analysis of risk factors of QoL in NPC patients. Similarly, univariable and multivariable logistic regression analysis was used for the analysis of risk factors of psychological resilience in NPC patients. Kaplan-Meier curves were generated to compare the overall survival rate and progression-free survival rate between the improved group and deteriorated group using the log-rank test. All statistical analysis was performed using SPSS 20.0 (IBM Corp., NY, USA) and $\mathrm{P}<0.05$ was considered as statistically different.

\section{Results}

NPC patients were prospectively recruited for this study between March 2017 and February 2019 and 316 patients were screened for inclusion during the study period. A total of 136 patients were excluded: (I) older than 80 years: 23 patients; (II) receiving multiple radiotherapy methods: 11 patients; (III) psychiatric disorders: 5 patients; (IV) refusing to participate: 86 patients; (V) severe organ dysfunction: 6 patients; and (VI) primary tumors in other 


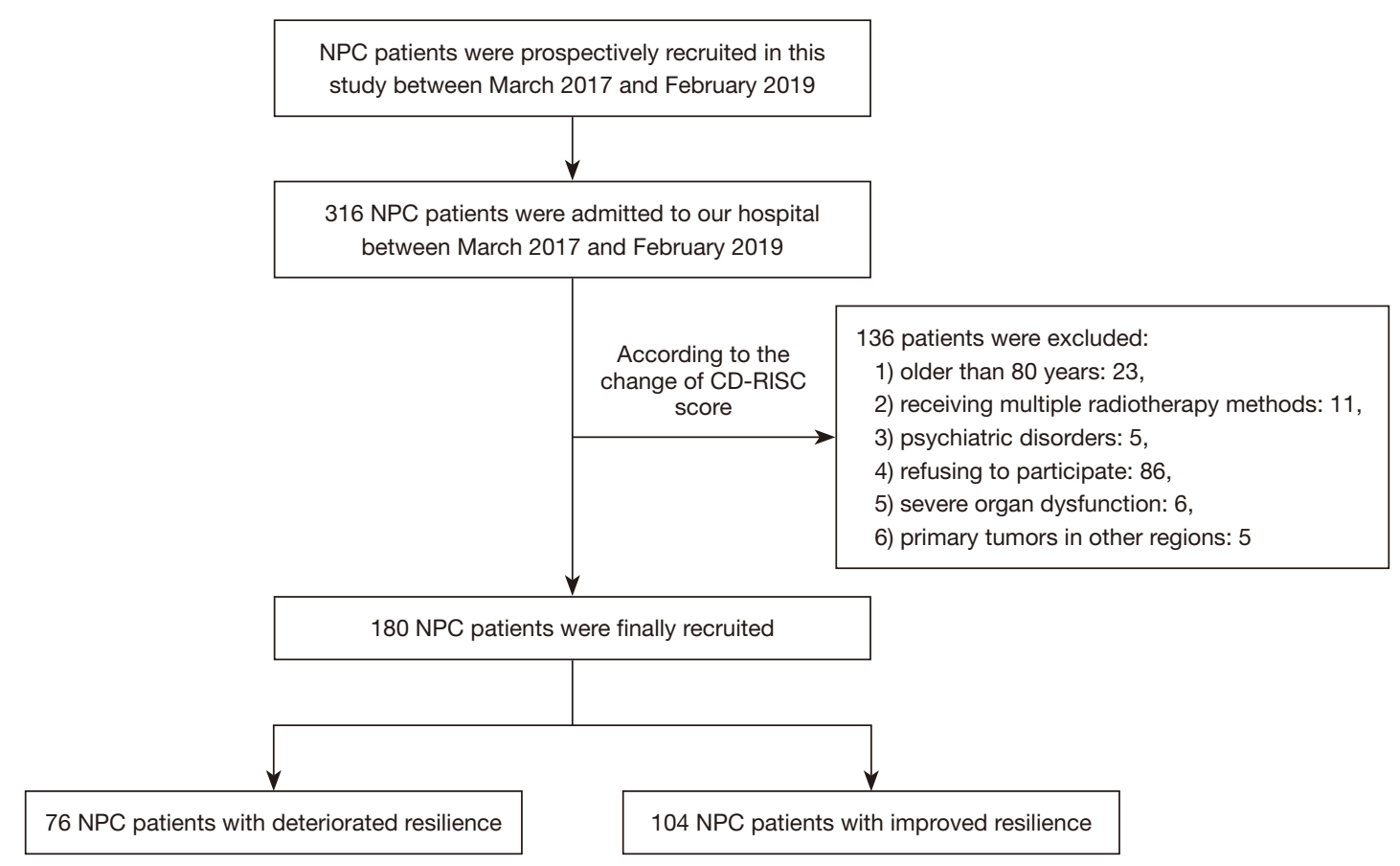

Figure 1 Flow chart of patient selection. NPC, nasopharyngeal carcinoma; CD-RISC, Connor-Davidson resilience scale.

regions: 5 patients. Finally, 180 patients were included in this study. Patients were then divided into the improved psychological resilience group, involving 104 patients, and the deteriorated psychological resilience group, involving 76 patients. The flow chart of this study is shown in Figure 1. The clinical and demographic data of the included patients are shown in Table 1. The mean age of the included patients was $49.0 \pm 9.8$ years and the mean BMI was $22.6 \pm 5.6$. There were more male patients $(\mathrm{n}=111)$ than female patients $(\mathrm{n}=69)$. About one fifth of all patients were single, divorced, or widowed and $28.3 \%$ of patients had a bachelor's degree or above. The histological type of all NPC cases was WHO type II or III and more than three fourths of NPC cases were WHO type III. The mean duration of disease was $21.5 \pm 6.5$ months. The clinical staging of most NPC cases was stage II or III. Forty patients $(22.2 \%)$ received extra chemotherapy for treatment. The mean dose of IMRT at the primary site was $67.6 \pm 4.7$ Gy and the mean fractional dose was $2.1 \pm 0.2$ Gy. Half of the included patients had a history of smoking and a small portion of patients had a history of chronic disease. The mean CD-RISC score before radiotherapy was $55.8 \pm 7.0$ points, the mean CDRISC score after radiotherapy was $58.4 \pm 7.8$ points, and the mean change of CD-RISC score was $2.6 \pm 4.0$ points.

Patients were divided into the improved group and deteriorated group (Table 2). Comparing the 2 groups, patients in the improved group were significantly younger than those in the deteriorated group $(\mathrm{P}=0.030)$ and more patients in the deteriorated group were single, divorced, or widowed $(\mathrm{P}=0.003)$. The educational level of patients in the improved group was higher than that in the deteriorated group $(\mathrm{P}=0.029)$. Additionally, there were more patients with stage II NPC and fewer patients receiving chemotherapy in the improved group. As for acute and late severe complications (Table 3), there were significantly more complications in the deteriorated group $(69.7 \%)$ than in the improved group (36.5\%). The most common complication was hearing loss $(32.2 \%)$, followed by headache $(21.1 \%)$ and acute mucositis (12.2\%). When determining the risk factors of psychological resilience in NPC patients with IMRT using multivariable logistic regression analysis (Table 4), it was found that older age, advanced stage of NPC, chemotherapy treatment, and the incidence of severe complications were important risk factors of psychological resilience, and married status may be a protective factor for psychological resilience.

Subsequently, the impact of psychological resilience on the QoL of NPC patients was analyzed. Firstly, the scores of 15 items in the QLQ-C30 and 13 items in the HN35 before radiotherapy were compared between the improved group and deteriorated group (Figure 2). No significant 
Table 1 Demographic and clinical characteristics of 180 non-distant NPC patients

\begin{tabular}{|c|c|}
\hline Variables & Number \\
\hline Age (year) & $49.0 \pm 9.8$ \\
\hline \multicolumn{2}{|l|}{ Gender, n (\%) } \\
\hline Male & $111(61.7)$ \\
\hline Female & 69 (38.3) \\
\hline BMI & $22.6 \pm 5.6$ \\
\hline \multicolumn{2}{|l|}{ Marital status, n (\%) } \\
\hline Married & $142(78.9)$ \\
\hline Single/divorced/widowed & $38(21.1)$ \\
\hline \multicolumn{2}{|l|}{ Educational level, n (\%) } \\
\hline High school or below & $129(71.7)$ \\
\hline Bachelor's degree or above & $51(28.3)$ \\
\hline \multicolumn{2}{|l|}{ Histological type, n (\%) } \\
\hline WHO type II & $42(23.3)$ \\
\hline WHO type III & $138(76.7)$ \\
\hline Disease duration (month) & $21.5 \pm 6.5$ \\
\hline \multicolumn{2}{|l|}{ Clinical staging, n (\%) } \\
\hline 1 & $11(6.1)$ \\
\hline II & $70(38.9)$ \\
\hline III & $68(37.8)$ \\
\hline IV & $31(17.2)$ \\
\hline \multicolumn{2}{|l|}{ Chemotherapy, n (\%) } \\
\hline None & $140(77.8)$ \\
\hline Induction chemotherapy & $16(8.9)$ \\
\hline Concurrent radiochemotherapy & $24(13.3)$ \\
\hline IMRT dose at primary site (Gy) & $67.6 \pm 4.7$ \\
\hline Fractional dose (Gy) & $2.1 \pm 0.2$ \\
\hline Smoking history, n (\%) & $94(52.2)$ \\
\hline \multicolumn{2}{|l|}{ History of chronic disease, n (\%) } \\
\hline Hypertension & $22(12.2)$ \\
\hline Diabetes mellitus & $13(7.2)$ \\
\hline Chronic heart disease & $6(3.3)$ \\
\hline Chronic lung disease & $5(2.8)$ \\
\hline Chronic kidney disease & $7(3.9)$ \\
\hline
\end{tabular}

Table 1 (continued)
Table 1 (continued)

\begin{tabular}{lc}
\hline Variables & Number \\
\hline Psychological resilience & \\
CD-RISC score before radiotherapy & $55.8 \pm 7.0$ \\
CD-RISC score after radiotherapy & $58.4 \pm 7.8$ \\
Change of CD-RISC score & $2.6 \pm 4.0$ \\
\hline NPC, nasopharyngeal carcinoma; BMI, body mass index; \\
WHO, World Health Organization; IMRT, intensity-modulated \\
radiotherapy; CD-RISC, Connor-Davidson resilience scale.
\end{tabular}

difference was found in QLQ-C30 scores and HN35 scores before radiotherapy between the 2 groups. Secondly, the scores of the QLQ-C30 and HN35 after radiotherapy were compared between the 2 groups (Figure 3). Contrary to the comparison of scores before radiotherapy, significant differences were found in most items in the QLQ-C30 and $\mathrm{HN} 35$ between the 2 groups, indicating that patients in the improved group had significantly higher QoL than those in the deteriorated group. Thirdly, the risk factors of QoL based on the global QoL score from the QLQ-C30 in NPC patients were determined using multivariable linear regression analysis (Table 5). Deteriorated psychological resilience was identified as an important risk factor of QoL in NPC patients. Additionally, older age, advanced stage of NPC, and the incidence of severe complications were important risk factors of QoL, and married status may play a protective role.

The mean follow-up duration of the included patients was $33.4 \pm 6.4$ months. Kaplan-Meier curves of overall survival and progression-free survival were generated as shown in Figure 4, and were compared between the improved group and deteriorated group. The results indicated that patients in the improved group had significantly higher overall survival and progression-free survival than those in the deteriorated group $(\mathrm{P}=0.010$ and 0.008$)$.

\section{Discussion}

To the best of our knowledge, this is the first study investigating the psychological resilience of NPC patients during treatment with IMRT. It was found that more patients had improved psychological resilience after IMRT. Older age, advanced stage of NPC, chemotherapy treatment, and the incidence of severe complications 
Table 2 Comparisons of demographic and clinical characteristics between NPC patients with deteriorated resilience and improved resilience

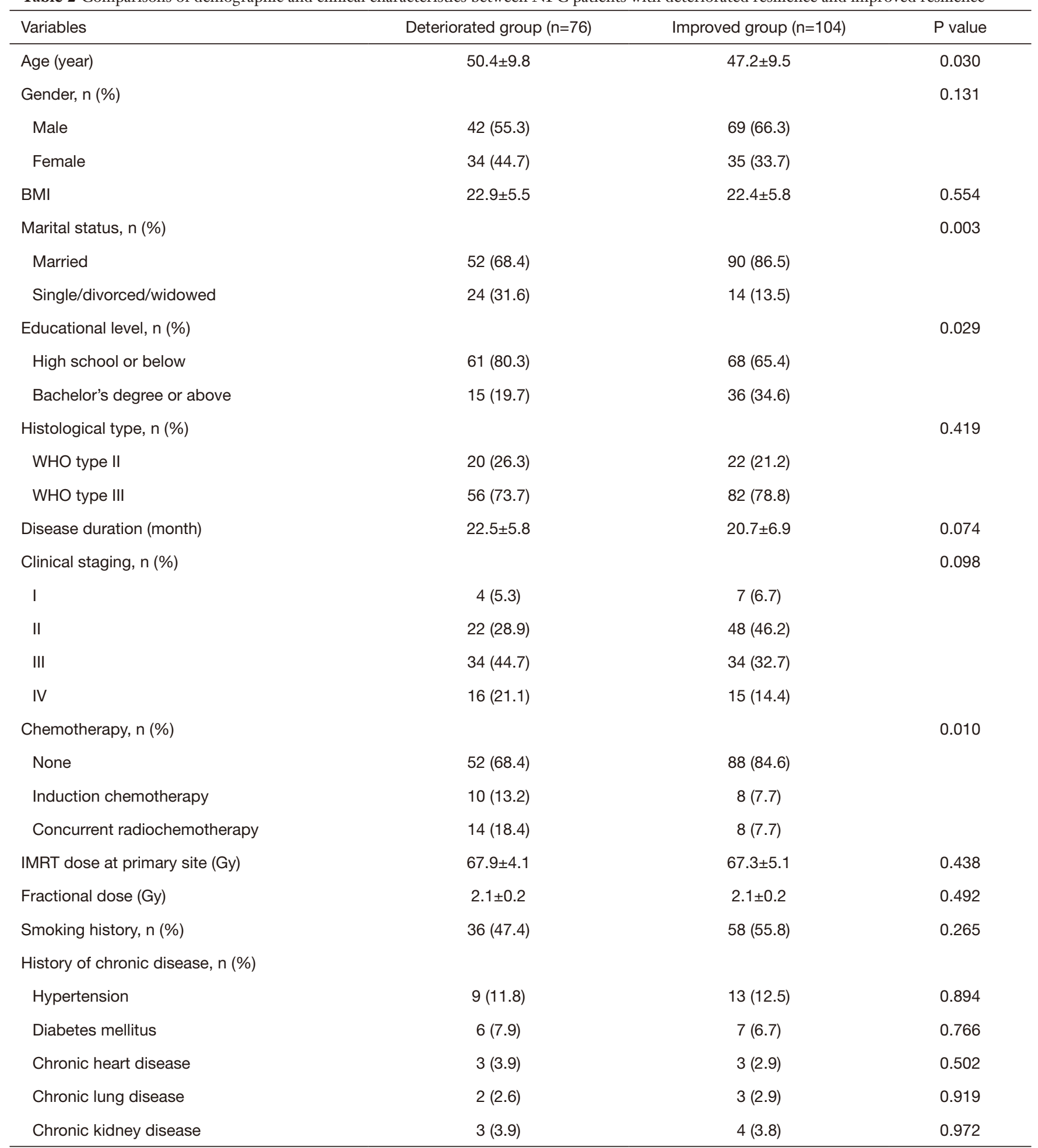

Table 2 (continued) 
Table 2 (continued)

\begin{tabular}{|c|c|c|c|}
\hline Variables & Deteriorated group $(n=76)$ & Improved group $(n=104)$ & $P$ value \\
\hline CD-RISC score before radiotherapy & $55.8 \pm 7.1$ & $55.7 \pm 6.9$ & 0.948 \\
\hline CD-RISC score after radiotherapy & $54.1 \pm 6.1$ & $61.5 \pm 7.5$ & $<0.001$ \\
\hline Change of CD-RISC score & $-1.7 \pm 1.7$ & $5.7 \pm 1.2$ & $<0.001$ \\
\hline
\end{tabular}

NPC, nasopharyngeal carcinoma; BMI, body mass index; WHO, World Health Organization; IMRT, intensity-modulated radiotherapy; CD-RISC, Connor-Davidson resilience scale.

Table 3 Acute and late severe complications in NPC patients

\begin{tabular}{|c|c|c|c|c|}
\hline Variables & Total $(n=180), n(\%)$ & Deteriorated group $(n=76), n(\%)$ & Improved group $(\mathrm{n}=104), \mathrm{n}(\%)$ & $P$ value \\
\hline Headache & $38(21.1)$ & $25(32.9)$ & $13(12.5)$ & 0.001 \\
\hline Acute mucositis & $22(12.2)$ & $15(19.7)$ & $7(6.7)$ & 0.004 \\
\hline Mucosal necrosis & $11(6.1)$ & $7(8.2)$ & $4(3.8)$ & 0.138 \\
\hline Cranial nerve palsy & $16(8.9)$ & $10(13.2)$ & $6(5.8)$ & 0.085 \\
\hline Temporal lobe necrosis & $3(1.7)$ & $3(3.9)$ & $0(0)$ & 0.074 \\
\hline Trismus & $15(8.3)$ & $9(11.8)$ & $6(5.8)$ & 0.145 \\
\hline Neck fibrosis & $5(2.8)$ & $2(2.6)$ & $3(2.9)$ & 0.919 \\
\hline
\end{tabular}

NPC, nasopharyngeal carcinoma.

Table 4 Risk factors of psychological resilience in NPC patients according to multivariable logistic regression analysis

\begin{tabular}{lccc}
\hline Variables & P value & OR & $0.95 \%$ Cl \\
\hline Older age & 0.014 & 0.954 & 0.991 \\
Female vs. male & 0.266 & 0.667 & 3.360 \\
Married vs. single/divorced/widowed & 0.015 & 3.003 & 2.105 \\
Bachelor's degree or above vs. high school or below & 0.064 & 1.044 & $0.959,4.608$ \\
Longer disease duration & 0.129 & 1.934 & $0.988,1.103$ \\
III/IV stage vs. I/II stage & 0.044 & 2.789 & $3.017,3.676$ \\
Chemotherapy vs. no chemotherapy & 0.020 & 3.684 & $1.178,6.604$ \\
Severe complication & $<0.001$ & $1.840,7.375$ \\
\hline
\end{tabular}

NPC, nasopharyngeal carcinoma. 

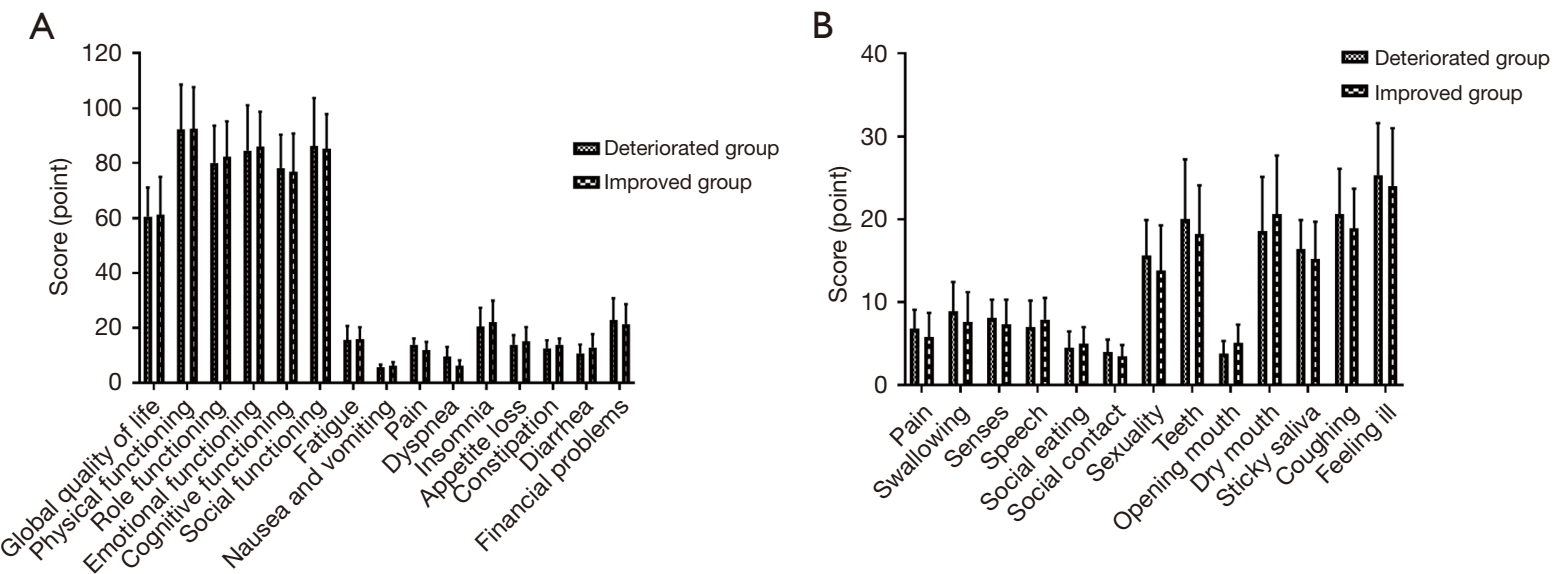

Figure 2 QLQ-C30 (A) and HN35 (B) scores in the improved group and deteriorated group before IMRT. QLQ-C30, Quality of Life Questionnaire-Core 30-questions; HN35, Head and Neck 35-questions; IMRT, intensity-modulated radiotherapy.
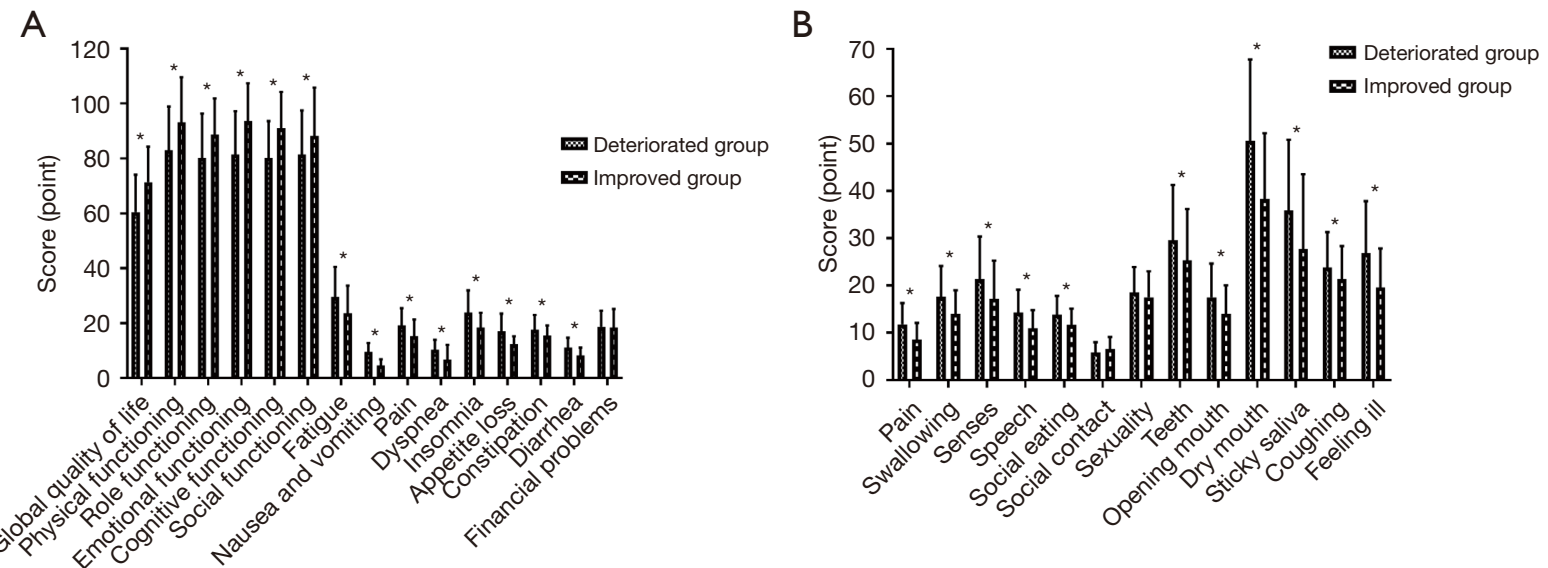

Figure 3 QLQ-C30 (A) and HN35 (B) scores in the improved group and deteriorated group 1 year after IMRT. QLQ-C30, Quality of Life Questionnaire-Core 30-questions; HN35, Head and Neck 35-questions; IMRT, intensity-modulated radiotherapy. *, P<0.05.

Table 5 Risk factors of QoL based on the QLQ-C30 in NPC patients according to multivariable linear regression analysis

\begin{tabular}{lccc}
\hline Variables & P value & Beta & $95 \%$ Cl \\
\hline Deteriorated psychological resilience vs. improved psychological resilience & $<0.001$ & -8.891 & $-12.770,-5.191$ \\
Older age & 0.024 & -0.174 & $-0.023,-0.325$ \\
Married vs. single/divorced/widowed & 0.006 & 4.765 & -2.591 \\
Bachelor's degree or above vs. high school or below & 0.131 & -3.426 & $-6.775,-0.077$ \\
III/IV stage vs. I/II stage & 0.045 & -0.093 & $-0.318,0.133$ \\
Longer disease duration & 0.418 & 1.610 & $-1.363,4.583$ \\
Chemotherapy vs. no chemotherapy & 0.287 & -7.657 & $-10.889,-4.425$ \\
Severe complication & $<0.001$ & & -5.775 \\
\hline
\end{tabular}

QoL, quality of life; QLQ-C30, Quality of Life Questionnaire-Core 30-questions; NPC, nasopharyngeal carcinoma. 
A

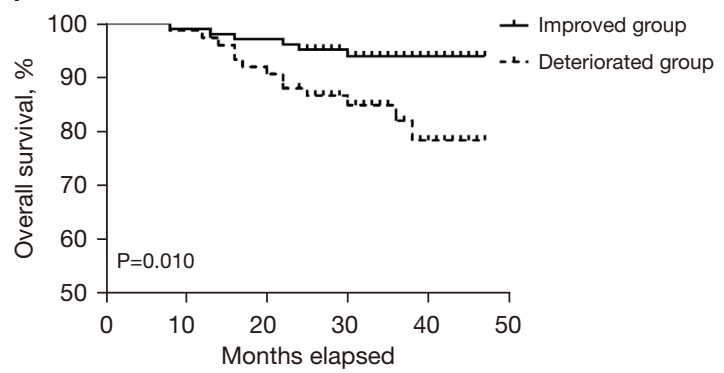

B

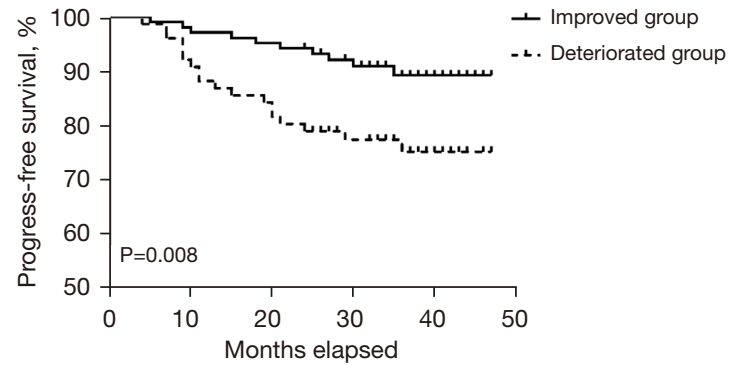

Figure 4 Overall survival (A) and progression-free survival (B) of the improved group and deteriorated group.

contributed to poor psychological resilience, and married status may be a protective factor for psychological resilience. Furthermore, deteriorated psychological resilience had a negative impact on QoL and decreased both the overall survival and progression-free survival of NPC patients. This study undertook a preliminary discussion on the current situation and influence of the psychological status of NPC patients, which will be expanded on in future studies.

The psychological resilience of cancer patients has always been a topic of discussion. A cross-sectional study in Pakistan showed that $8 \%$ of patients with head and neck cancer have severe depression and 3\% have severe anxiety (16). The emergence of depression and anxiety could significantly reduce the prognosis of patients (16). In another Chinese study, NPC patients often experienced a certain degree of depression and anxiety in the early stage of radiotherapy. Similar findings have been shown in some other cancers. The psychological resilience of patients with advanced lung cancer was closely related to patients' QoL $(17,18)$. Depressive symptoms were very common in patients with prostate cancer and could affect the patient's willingness to live (19). In our study, the psychological resilience of NPC patients improved to some extent within 1 year after receiving IMRT treatment, and the average CD-RISC score increased from $55.8 \pm 7.0$ to $58.4 \pm 7.8$ within the 1 -year period. In the detailed analysis, we found that the psychological resilience of 104 patients after treatment was improved to varying degrees compared with that before treatment, accounting for $57.8 \%$ of the total cases. However, the psychological resilience of the remaining $42.2 \%$ of patients decreased significantly. This suggest that, in general, the psychological resilience of NPC patients after IMRT treatment is on the rise, but the deterioration of individual patients' psychological status should be paid attention to. According to our results, the risk factors of poor psychological resilience mainly included older age, advanced stage of NPC, the use of chemotherapy, and the occurrence of severe complications. Elderly patients are psychologically fragile and tend to have negative emotions when unexpected events occur. The occurrence of severe complications is related to the dose of radiotherapy, the use of chemotherapy, and individual tolerance. In clinical practice, more attention should be paid to the mental health of patients with severe complications, providing appropriate psychological counseling, and adjusting the psychological resilience of patients.

The occurrence of complications is one of the most important problems in NPC patients receiving radiotherapy. Our study recorded the occurrence of some severe complications. The results showed that the total incidence of complications was $50.6 \%$, of which hearing loss, severe headache, and acute mucositis were the most common complications, with an incidence of more than $10 \%$. The follow-up analysis showed that the occurrence of severe complications will affect the patient's psychological resilience and QoL at the same time. In previous studies, there were some other complications not recorded in our study. Kong et al. showed that massive nasopharynx hemorrhage was one of the major serious complications in NPC patients, which can lead to death in severe cases (20). It was also reported that the probability of osteoradionecrosis in NPC patients after radiotherapy is significantly underestimated (21). Once osteoradionecrosis occurs, surgery is the main treatment, which will significantly affect the QoL of patients. In addition, nausea and vomiting are the most common complications of NPC patients after radiotherapy. It is reported that the incidence of nausea and vomiting can be as high as more than $80 \%$ (22-25). However, the symptoms of nausea and vomiting are mostly mild and relatively easy to treat, thus they were 
not recorded in our study. In general, the complications of NPC patients after radiotherapy have a significant impact on the prognosis of patients, and they should be actively dealt with and carefully cared for.

There are relatively few studies on QoL in NPC patients. Zheng et al. showed that IMRT can reduce complications, improve short-term efficacy, and improve QoL in patients with NPC compared with 3D conformal radiotherapy (26). Zheng et al. showed that high-quality nursing intervention can more effectively improve patients' satisfaction and QoL (26). However, few studies have explored the effect of psychological factors on QoL in NPC patients. One previous study showed that psychological distress was negatively correlated with QoL, but the evaluation of psychological distress was rough because only psychological distress thermometer was used to assess the level of psychological distress (27). Our study is the first to examine the correlation between psychological resilience and patients' QoL through mature scales, which lays a preliminary foundation for future research.

Nowadays, the prognosis of NPC patients after radiotherapy has been greatly improved. Wang et al. found that the 15 -year overall survival rate of early NPC patients treated with IMRT was $93.3 \%$ (28). In patients with advanced NPC, the 5-year overall survival rate can also reach $82.5 \%$ (29). In our study, the prognosis of patients was also relatively good, with a 3 -year overall survival rate of $89.4 \%$. However, there was a significant difference in the overall survival rate between the improved group and the deteriorated group, suggesting that psychological resilience played an important role in the long-term outcomes of NPC patients after treatment.

Some limitations should be noted in this study. Firstly, this study is a single center prospective study. The results of this study may therefore only be applicable to the patients included in this study. There are still some questions about whether the results of this study can be directly applied to patients in other centers. Secondly, the operation of IMRT in this study was performed by the radiotherapy department, and our department was unable to alter the radiation dose used in the treatment. Radiation dose may have a great impact on the prognosis and severe complications of patients, although there was no significant difference in the radiation dose between the 2 groups at baseline. Thirdly, the CD-RISC was used to evaluate the psychological resilience of patients in this study, but this scale may not fully express the psychological status of patients. However, filling in other scales may make patients feel annoyed, which may reduce compliance. This is a dilemma that needs to be solved with a better scale in the future.

\section{Conclusions}

This study identified the important role of psychological resilience in the prognosis of NPC patients during treatment with IMRT. This highlights that more attention should be paid to the psychological status of NPC patients during treatment with IMRT, the timely detection of the negative psychological status of patients, and improving the psychological resilience of patients. This study also provides more evidence for the importance of psychological care of NPC patients.

\section{Acknowledgments}

Funding: None.

\section{Footnote}

Reporting Checklist: The authors have completed the STROBE reporting checklist. Available at https://apm. amegroups.com/article/view/10.21037/apm-21-3592/rc

Data Sharing Statement: Available at https://apm.amegroups. com/article/view/10.21037/apm-21-3592/dss

Conflicts of Interest: All authors have completed the ICMJE uniform disclosure form (available at https://apm. amegroups.com/article/view/10.21037/apm-21-3592/coif). The authors have no conflicts of interest to declare.

Ethical Statement: The authors are accountable for all aspects of the work in ensuring that questions related to the accuracy or integrity of any part of the work are appropriately investigated and resolved. All procedures performed in this study involving human participants were in accordance with the Declaration of Helsinki (as revised in 2013). The study was approved by institutional review board of the Affiliated Hospital of Jiangnan University (No. 2016033) and informed consent was taken from all the patients.

Open Access Statement: This is an Open Access article distributed in accordance with the Creative Commons Attribution-NonCommercial-NoDerivs 4.0 International License (CC BY-NC-ND 4.0), which permits the non- 
commercial replication and distribution of the article with the strict proviso that no changes or edits are made and the original work is properly cited (including links to both the formal publication through the relevant DOI and the license). See: https://creativecommons.org/licenses/by-nc-nd/4.0/.

\section{References}

1. Zou J, Sun Q, Akiba S, et al. A case-control study of nasopharyngeal carcinoma in the high background radiation areas of Yangjiang, China. J Radiat Res 2000;41 Suppl:53-62.

2. Wen DW, Lin L, Mao YP, et al. Normal tissue complication probability (NTCP) models for predicting temporal lobe injury after intensity-modulated radiotherapy in nasopharyngeal carcinoma: A large registry-based retrospective study from China. Radiother Oncol 2021;157:99-105.

3. Chen MY, Chen YS, Hu LJ, et al. The end-of-treatment telephone response and prognosis of post-radiotherapy nasopharyngeal carcinoma patients in southern China. Int J Clin Exp Med 2015;8:16564-70.

4. Zheng L, Xu Q, Yang Z, et al. The effect of combined TPF and intensity modulated radiotherapy after TPF induction chemotherapy on locally advanced nasopharyngeal carcinoma: a retrospective analysis. Ann Palliat Med 2021;10:9669-77.

5. Li R, Su L, Hua Y, et al. Anxiety and depression status prior to radiotherapy in patients with nasopharyngeal carcinoma and its effect on acute radiation toxicities. Eur J Cancer Care (Engl) 2021;30:e13487.

6. Zhang YM, Kang YF, Zeng JJ, et al. Surface-Based Falff: A Potential Novel Biomarker for Prediction of Radiation Encephalopathy in Patients With Nasopharyngeal Carcinoma. Front Neurosci 2021;15:692575.

7. Pan XB, Huang ST, Chen KH, et al. Concurrent chemoradiotherapy degrades the quality of life of patients with stage II nasopharyngeal carcinoma as compared to radiotherapy. Oncotarget 2017;8:14029-38.

8. Poon DMC, Kam MKM, Johnson D, et al. Durability of the parotid-sparing effect of intensity-modulated radiotherapy (IMRT) in early stage nasopharyngeal carcinoma: A 15-year follow-up of a randomized prospective study of IMRT versus two-dimensional radiotherapy. Head Neck 2021;43:1711-20.

9. Moon SH, Cho KH, Lee CG, et al. IMRT vs. 2D-radiotherapy or 3D-conformal radiotherapy of nasopharyngeal carcinoma : Survival outcome in a Korean multi-institutional retrospective study (KROG 11-06). Strahlenther Onkol 2016;192:377-85.

10. Du T, Xiao J, Qiu Z, et al. The effectiveness of intensitymodulated radiation therapy versus $2 \mathrm{D}$-RT for the treatment of nasopharyngeal carcinoma: A systematic review and meta-analysis. PLoS One 2019;14:e0219611.

11. Mao YP, Zhou GQ, Liu LZ, et al. Comparison of radiological and clinical features of temporal lobe necrosis in nasopharyngeal carcinoma patients treated with $2 \mathrm{D}$ radiotherapy or intensity-modulated radiotherapy. $\mathrm{Br} \mathrm{J}$ Cancer 2014;110:2633-9.

12. Zhang B, Mo Z, Du W, et al. Intensity-modulated radiation therapy versus $2 \mathrm{D}-\mathrm{RT}$ or $3 \mathrm{D}$-CRT for the treatment of nasopharyngeal carcinoma: A systematic review and meta-analysis. Oral Oncol 2015;51:1041-6.

13. Bišof V, Rakušić Z, Bibić J, et al. Comparison of intensity modulated radiotherapy with simultaneous integrated boost (IMRT-SIB) and a 3-dimensional conformal parotid gland-sparing radiotherapy (ConPas 3D-CRT) in treatment of nasopharyngeal carcinoma: a monoinstitutional experience. Radiol Med 2018;123:217-26.

14. Lee PW, Kwan TT, Kwong DL, et al. A prospective study of the impact of nasopharyngeal cancer and radiotherapy on the psychosocial condition of Chinese patients. Cancer 2007;109:1344-54.

15. McDowell LJ, Rock K, Xu W, et al. Long-Term Late Toxicity, Quality of Life, and Emotional Distress in Patients With Nasopharyngeal Carcinoma Treated With Intensity Modulated Radiation Therapy. Int J Radiat Oncol Biol Phys 2018;102:340-52.

16. Zahid N, Zahid W, Khalid W, et al. Resilience and its associated factors in head and neck cancer patients in Pakistan: an analytical cross-sectional study. BMC Cancer 2021;21:888.

17. Wang H, Yue H, Ren M, et al. Dyadic effects of familyfunctioning and resilience on quality of life in advanced lung cancer patients and caregivers: An actor-partner interdependence mediation model. Eur J Oncol Nurs 2021;52:101963.

18. Chen S, Mei R, Tan C, et al. Psychological resilience and related influencing factors in postoperative nonsmall cell lung cancer patients: A cross-sectional study. Psychooncology 2020;29:1815-22.

19. Zhao X, Sun M, Yang Y. Effects of social support, hope and resilience on depressive symptoms within 18 months after diagnosis of prostate cancer. Health Qual Life Outcomes 2021;19:15.

20. Kong F, Zhou J, Du C, et al. Long-term survival and 
late complications of intensity-modulated radiotherapy for recurrent nasopharyngeal carcinoma. BMC Cancer 2018;18:1139.

21. Huang WB, Wong STS, Chan JYW. Role of surgery in the treatment of osteoradionecrosis and its complications after radiotherapy for nasopharyngeal carcinoma. Head Neck 2018;40:369-76.

22. Yin WJ, Chen DP, Wang MY, et al. Prognostic value of hypertension in patients with nasopharyngeal carcinoma treated with intensity-modulated radiation therapy. Ann Transl Med 2021;9:1313.

23. Yang H, Liu C, Luo S, et al. Prognostic analysis of 152 patients with distant metastasis after intensity-modulated radiotherapy for nasopharyngeal carcinoma. Ann Palliat Med 2021;10:6824-32.

24. You R, Liu YP, Huang PY, et al. Efficacy and Safety of Locoregional Radiotherapy With Chemotherapy vs Chemotherapy Alone in De Novo Metastatic Nasopharyngeal Carcinoma: A Multicenter Phase 3 Randomized Clinical Trial. JAMA Oncol 2020;6:1345-52.

25. Wu P, Zhao Y, Xiang L, et al. Management of Chemotherapy for Stage II Nasopharyngeal Carcinoma in the Intensity-Modulated Radiotherapy Era: A Review. Cancer Manag Res 2020;12:957-63.

Cite this article as: $\mathrm{Lu} \mathrm{N}$, Qin T, Hu W. Impact of changes in psychological resilience during treatment with intensitymodulated radiotherapy on nasopharyngeal carcinoma patients: a prospective study. Ann Palliat Med 2022;11(1):123-134. doi: 10.21037/apm-21-3592
26. Zheng L, Tong L, Du F, et al. Effect of three-dimensional conformal radiotherapy and intensity-modulated radiotherapy on parotid gland function and quality of life in patients with nasopharyngeal carcinoma. Am J Transl Res 2021;13:5272-9.

27. Wang X, Lv Y, Li W, et al. Correlation between Psychosocial Distress and Quality of Life in Patients with Nasopharyngeal Carcinoma following Radiotherapy. J Oncol 2018;2018:3625302.

28. Wang L, Miao J, Huang H, et al. Long-term Survivals, Toxicities and the Role of Chemotherapy in EarlyStage Nasopharyngeal Carcinoma Patients Treated with Intensity-modulated Radiation Therapy: A Retrospective Study with 15-year Follow-up. Cancer Res Treat 2022;54:118-29.

29. Fangzheng W, Chuner J, Haiyan Q, et al. Survival without concurrent chemotherapy for locoregionally advanced nasopharyngeal carcinoma treated with induction chemotherapy plus intensity-modulated radiotherapy: Single-center experience from an endemic area. Medicine (Baltimore) 2019;98:e18484.

(English Language Editor: C. Betlzar) 